\title{
Depression Anxiety and Stress Scale (DASS-21) - Short Form: Adaptação e Validação para Adolescentes Brasileiros
}

\author{
Naiana Dapieve Patias - Universidade Federal do Rio Grande do Sul e Faculdade Meridional (IMED), Passo Fundo, Brasil \\ Wagner De Lara Machado - Pontifícia Universidade Católica de Campinas, Campinas, Brasil \\ Denise Ruschel Bandeira - Universidade Federal do Rio Grande do Sul, Porto Alegre, Brasil \\ Débora Dalbosco Dell'Aglio - Universidade Federal do Rio Grande do Sul, Porto Alegre, Brasil
}

\begin{abstract}
Resumo
Este estudo teve como objetivo adaptar e validar a Depression Anxiety and Stress Scale (DASS-21) - Short Form para adolescentes brasileiros. Participaram do estudo 426 adolescentes de 12 a 18 anos $(M=14,91 ; D P=1,66)$, sendo 264 meninas $(62 \%)$ e 162 meninos (39\%), de escolas públicas da cidade de Porto Alegre-RS, que responderam a uma versão adaptada, nomeada Escala de Depressão, Ansiedade e Estresse para Adolescentes (EDAE-A). As subescalas demonstraram níveis adequados de consistência interna, variando entre 0,83 e 0,90 . A análise fatorial confirmatória indicou que o melhor modelo foi o de três fatores, confirmando o modelo original com as dimensões depressão, ansiedade e estresse. No escore total e por fatores, houve diferença por sexo, com maior média entre as meninas em todos os escores. Conclui-se que a EDAE-A apresenta qualidades psicométricas favoráveis, mostrando-se um simples e adequado instrumento de levantamento de sintomas de depressão, ansiedade e estresse para adolescentes brasileiros.

Palavras-chave: adolescentes, depressão, ansiedade, estresse, DASS-21, EDAE-A
\end{abstract}

\section{Depression Anxiety and Stress Scale (DASS-21) - Short Form: Adaptation and Validation for Brazilian Adolescents}

\begin{abstract}
This study aimed to adapt and validate the short form of the Depression Anxiety and Stress Scale (DASS-21) for Brazilian adolescents. The participants were 426 adolescents aged 12 to 18 years $(M=14.91, S D=1.66), 264$ girls $(62 \%)$ and 162 boys (39\%), of public schools in the city of Porto Alegre-RS, who answered an adapted version named Escala de Depressão, Ansiedade e Estresse para Adolescentes (EDAE-A). The subscales showed suitable levels of internal consistency, ranging between 0.83 and 0.90 . Confirmatory factor analysis indicated that the best model was the three factors confirming the original model with depression, anxiety and stress dimensions. In the total score and factors, there were differences by sex, with highest average to girls in all scores. We concluded that the EDAE-A presents favorable psychometric qualities, being a simple and suitable survey instrument for identifying symptoms of depression, anxiety and stress in Brazilian adolescents.

Keywords: adolescent, depression, anxiety, stress, DASS-21, EDAE-A
\end{abstract}

\section{Depression Anxiety and Stress Scale (DASS-21) - Short Form:} Adaptación y Validación para los Adolescentes Brasileños

\begin{abstract}
Resumen
Este estudio tuvo como objetivo adaptar y validar la Depression Anxiety and Stress Scale (DASS-21) - Short Form para adolescentes brasileños. Participaron del estudio 426 adolescentes entre 12 y 18 años $(M=14,91, S D=1,66)$, siendo 264 de sexo femenino (62\%) y 162 de sexo masculino (39\%), de escuelas públicas de la ciudad de Porto Alegre-RS, que respondieron a una versión adaptada nominada Escala de Depresión, Ansiedad y Estrés para Adolescentes (EDAE-A). Las sub-escalas demostraron niveles adecuados de consistencia interna, oscilando entre 0,83 y 0,90 . El análisis factorial confirmatorio indicó que el mejor modelo fue el de los tres factores, lo que confirma el modelo original con las dimensiones depresión, ansiedad y estrés. En el resultado total y por factores hubo diferencia con relación al sexo, con mayores medias entre el sexo femenino en todos los resultados. La EDAE-A presenta cualidades psicométricas favorables, siendo un simple y adecuado instrumento de levantamiento de síntomas de depresión, ansiedad y estrés para adolescentes brasileños.

Palabras-clave: adolescentes, depresión, ansiedad, estrés, DASS-21, EDAE-A
\end{abstract}

\section{Introdução}

A depressão é o principal fator de risco para o suicídio, sendo a segunda maior causa de morte na população adolescente (Thapar, Collinshaw, Pine, \& Thapar, 2012). No entanto, esse grupo etário tem recebido pouca atenção em relação à saúde mental (Brasil, 2010). Segundo a World Health Organization
(2012), alguns transtornos mentais têm seu início marcado na infância e pré-adolescência. Estudos recentes têm identificado a origem de muitas doenças mentais nesse período, principalmente a depressão, com alta prevalência na população adolescente, e comorbidades associadas, tais como os transtornos de ansiedade, que chegam a ser encontrados em $30 \%$ a $80 \%$ dos casos (Bahls \& Bahls, 2002; Caspi et al., 2014; Jatobá \& 
Bastos, 2007; Patrick, Dyck, \& Bramston, 2010; Thapar et al., 2012). Além disso, apresentar um transtorno como a depressão na adolescência está associado a uma gama de transtornos mentais na vida adulta, tais como a ansiedade, desordens relacionadas ao abuso de substâncias psicoativas, bipolaridade e comportamento suicida (Thapar et al., 2012). De fato, segundo Caspi et al. (2014), os transtornos mentais são positivamente correlacionados de tal forma que, apresentar um transtorno mental, aumenta a probabilidade de desenvolver outro no futuro.

Autores (Hess \& Falcke, 2013; Pinto et al., 2014; Rocha, Zeni, Caetano, \& Kieling, 2013) ressaltam que as modificações biopsicossociais da adolescência podem predispor à ocorrência de transtornos mentais, aumentando a chance de episódios depressivos de cinco para $20 \%$. Além disso, aspectos hormonais podem tornar os adolescentes mais sensíveis a eventos estressores do ambiente, o que seria uma das explicações para o aumento dos episódios depressivos nesse período.

Estima-se que, no mundo, a cada ano, 20\% dos adolescentes apresentam um transtorno mental que pode levar ao suicídio (WHO, 2012). No Brasil, revisão de literatura realizada por Assis, Avanci, Pesce e Ximenes (2009) indicou uma prevalência que varia de 8 a 51\%, segundo a escala Self Report Questionnarie (SRQ20) que mapeia sintomas de transtornos mentais, tais como depressão e ansiedade. Outros estudos brasileiros sugerem que as taxas de transtornos mentais em adolescentes variam de 10 a 20\% (Kieling \& Belter, 2012).

O campo da saúde mental na adolescência é relativamente novo, se comparado a outros campos da Psiquiatria. Por exemplo, até 1970, acreditava-se que a depressão em adolescentes fosse rara (Bahls \& Bahls, 2002). Além disso, há no Brasil, uma escassez de serviços dedicados à promoção de saúde mental de crianças e adolescentes, especialmente no setor público (Rocha et al., 2013).

Outros aspectos dificultam o diagnóstico adequado, tais como a dificuldade em identificar a depressão na adolescência, pois muitos sintomas confundem-se com comportamentos próprios do adolescente. Além disso, nem sempre a depressão, nesse período de vida, manifesta-se da mesma forma que em adultos, embora sejam utilizados os mesmos sintomas para o diagnóstico (American Psychiatric Association - APA, 2014; Argimon, Terroso, Barbosa, \& Lopes, 2013; Bahls \& Bahls, 2002; Rocha et al., 2013). Segundo Bahls e Bahls (2002), geralmente na adolescência, podem predominar sintomas de irritabilidade e instabilidade, ao invés da queixa de tristeza. Além disso, é comum a perda de energia, apatia, desinteresse, sentimentos de desesperança e culpa, perturbações do sono, isolamento e dificuldade de concentração.

Outro aspecto que dificulta o diagnóstico e tratamento é que os sintomas de internalização, voltados para o próprio indivíduo e que incluem sintomas de ansiedade, depressão e somatização (APA, 2014), tendem a ser mais reportados pelos próprios pacientes do que pelos professores e cuidadores (Rocha et al., 2013). No entanto, nem sempre os adolescentes reconhecem os sintomas da doença (Rosa, Loureiro, \& Sequeira, 2014) ou, quando identificam, por constrangimento e pelo estigma social associado à doença, podem não procurar ajuda, preferindo pensar que podem resolver sozinhos. Quando buscam ajuda, geralmente é por meio dos amigos e não de um profissional (Gulliver, Griffiths, \& Christensen, 2010; Rosa et al., 2014).

Assim, a identificação do transtorno, principalmente de forma precoce e acurada é requisito essencial que pode auxiliar no processo de identificação do sofrimento mental e até mesmo de um possível diagnóstico - junto com outros instrumentos de avaliação - para posterior tratamento e prognóstico. A falta de instrumentos específicos para a população adolescente dificulta o diagnóstico. Além disso, é comum, no Brasil, que a avaliação de sintomas de depressão, ansiedade e estresse seja realizada de forma independente, e não em conjunto.

Este estudo teve como objetivo adaptar e validar a Depression, Anxiety and Stress Scale - Short Form (DASS21) para adolescentes brasileiros, um instrumento de mapeamento de sintomas de depressão, ansiedade e estresse. A escolha desse instrumento justifica-se por envolver um modelo teórico que discrimina bem sintomas de ansiedade e depressão, nem sempre diferenciados por outras escalas ou instrumentos (Lovibond \& Lovibond, 1995; Pais-Ribeiro, Honrado, \& Leal, 2004). Foi traduzida e adaptada para diversas faixas etárias e em diversos países, tais como: Portugal (Apóstolo, Mendes, \& Azeredo, 2006; Pais-Ribeiro et al., 2004; Leal, Antunes, Passos, Pais-Ribeiro, \& Maroco 2009), Brasil (Machado \& Bandeira, 2013; Vignola \& Tucci, 2014), Espanha (Daza, Novy, Stanley, \& Averill, 2002), Grécia (Lyrakos, Arvaniti, Smyrnioti, \& Kostopanagiotou, 2011), Turquia (Akin \& Çetin, 2007), Malásia (Musa, Fadzil, \& Zain, 2007), entre outros países. Além disso, a escala é composta por poucos itens, sendo de fácil e rápida administração. 


\section{Método}

\section{Participantes}

Participaram 426 adolescentes, com idades entre 12 e 18 anos $(M=14,91 ; D P=1,66), 264$ meninas (62\%) e 162 meninos (39\%), de cinco escolas públicas de periferias da cidade de Porto Alegre (RS, Brasil), estudantes do $7^{\circ}$ ano do Ensino Fundamental ao $3^{\circ}$ ano do Ensino Médio. As escolas foram escolhidas por conveniência segundo possibilidades e aceite das instituições. O número de participantes foi estimado por meio de cálculo amostral a partir do número total de alunos de Ensino Fundamental e Ensino Médio matriculados em escolas públicas de Porto Alegre - RS, com uma margem de erro estabelecida de 5\% (previsão em torno de 400 adolescentes).

\section{Instrumentos}

A Depression, Anxiety and Stress Scale - Short Form (DASS-21) foi desenvolvida por Lovibond e Lovibond (1995) com o objetivo de medir e diferenciar, ao máximo, os sintomas de ansiedade e depressão. A escala é baseada no modelo tripartido em que os sintomas de ansiedade e depressão se agrupam em três estruturas básicas. Uma primeira, (a) definida pela presença de afeto negativo, como humor deprimido, insônia, desconforto e irritabilidade, que são sintomas inespecíficos e estão incluídos tanto na depressão como na ansiedade; a segunda engloba (b) fatores que constituem estruturas que representam sintomas específicos para depressão (anedonia, ausência de afeto positivo); por fim, a última estrutura refere-se aos (c) sintomas específicos de ansiedade (tensão somática e hiperatividade) (Watson et al., 1995).

Originalmente, a DASS foi construída e avaliada para população adulta, consistindo em 42 itens que refletem sintomas emocionais negativos. Os resultados de uma série de análises fatoriais durante o seu desenvolvimento indicaram que os principais sintomas de ansiedade incluem excitação fisiológica (mãos suadas, tremores, aumento do batimento cardíaco), bem como a consciência subjetiva do afeto ansioso e tendências de fuga ou evitação. Já os sintomas de depressão são: baixo afeto positivo, desesperança, desvalorização da vida, depreciação e inércia. Além disso, foi incluída, na versão original, uma subescala de tensão/esforço (Lovibond \& Lovibond, 1995). Esta última subescala surgiu empiricamente durante o desenvolvimento da subescala de depressão e de ansiedade, por meio de uma agregação de itens, como dificuldade em relaxar, tensão, impaciência, irritabilidade e agitação (Szabó, 2010).

Vários pesquisadores têm demonstrado que o DASS, tanto em sua versão original de 42 itens quanto em sua versão breve de 21 itens, é uma medida válida e confiável de depressão, ansiedade e tensão/estresse em populações clínica e não clínica de adultos, de várias culturas e etnias. Essa última versão é composta por itens da versão original selecionados conforme critérios de conteúdo, i.e., representação das subfacetas dos construtos avaliados, e estatísticos (carga fatorial; descrito em Lovibond \& Lovibond, 1995). Além disso, pesquisas com DASS-21 foram realizadas em amostras de crianças, adolescentes e jovens portugueses (Leal, Antunes, Passos, Pais-Ribeiro, \& Maroco, 2009; Pais-Ribeiro et al., 2004) e australianos (Szabó, 2010; Tully, Zajac, \& Venning, 2009), demonstrando propriedades psicométricas aceitáveis. No Reino Unido, Henry e Crawford (2005) reportaram medidas de fidedignidade variando de 0,82 a 0,90 para as três subescalas, e de 0,93 para a escala global, utilizada com adultos. No Brasil, a DASS21 foi adaptada e validada para 686 adultos, de diversas regiões do país, com média de idade de 33,88 (DP = 11,26), apresentando medidas de fidedignidade de 0,92 a 0,96 para as subescalas (Machado \& Bandeira, 2013). Os autores Vignola e Tucci (2014) também tiveram evidências de validade de 0,86 a 0,92 para uma amostra clínica de adultos paulistas $(M=39,9 ; D P=12,4), 62 \%$ mulheres. No entanto, ainda não foram desenvolvidos no Brasil estudos de validação para o uso da escala com adolescentes.

A versão da DASS-21 utilizada para este estudo foi uma adaptação da versão utilizada para adultos brasileiros por Machado e Bandeira (2013), mantendo as três subescalas para avaliar sintomas de depressão, ansiedade e estresse. Na DASS-21, os participantes indicam o grau em que experimentam cada um dos sintomas descritos nos itens durante a última semana (semana anterior), em uma escala do tipo Likert de 4 pontos entre 0 (não se aplica a mim) e 3 (aplica-se muito a mim, ou a maior parte do tempo). Pontuações para depressão, ansiedade e estresse são determinadas pela soma dos escores dos 21 itens. A estrutura fatorial da DASS-21 é estável e possui validade convergente e discriminante aceitáveis (Lovibond \& Lovibond, 1995).

Além da DASS-21, aplicou-se um questionário de dados sociodemográficos que foi construído especialmente para este estudo e que investiga informações sobre idade, sexo, escolaridade dos pais, configuração familiar, entre outros aspectos. 


\section{Procedimentos}

A pesquisa da qual se originou o banco de dados para este estudo foi aprovada por Comitê de Ética em Psicologia, CAAE (22080914.1.0000.5334) e seguiu os aspectos éticos que garantem a integridade dos participantes, de acordo com legislações brasileiras, tendo como base a Resolução no 466 (Brasil, 2012), e o Estatuto da Criança e do Adolescente (Brasil, 1990). Os pais dos adolescentes assinaram o Termo de Consentimento Livre e Esclarecido (TCLE), e os adolescentes deram seu assentimento, concordando com a participação no estudo.

Os procedimentos para adaptação e validação da escala foram os sugeridos por Borsa, Damásio e Bandeira (2012). Primeiramente, foi administrada uma avaliação qualitativa do instrumento. Para a primeira avaliação da escala foi selecionada uma escola por conveniência, convidando adolescentes de diferentes turmas e diferentes idades para participar do estudo. Essa primeira etapa teve por objetivo verificar os itens da DASS-21, se suas instruções e os termos presentes nas afirmativas dos itens estavam claros e compreensíveis. Dessa forma, aplicou-se o instrumento em 20 adolescentes entre 16 a 18 anos, de ambos os sexos, estudantes do primeiro ano do ensino médio de uma escola pública da cidade de Porto Alegre - RS. A aplicação foi coletiva, por duas psicólogas treinadas para esse procedimento. Juntamente com a escala, foi aplicado o instrumento de dados sociodemográficos. Durante a aplicação, as psicólogas circularam pela sala de aula a fim de verificar dúvidas dos adolescentes decorrentes das instruções e itens da escala. Após a aplicação, houve uma discussão coletiva sobre o entendimento e dificuldades dos adolescentes acerca das instruções e de cada item da escala. Verificou-se, no geral, que os adolescentes compreenderam os itens, embora tenham sugerido algumas modificações para melhor entendimento. As modificações sugeridas foram analisadas por três profissionais com experiência em instrumentos psicológicos. Houve consenso quanto a realizar as modificações, não necessitando de retrotradução, já que a escala já havia sido traduzida para o português (versão Machado \& Bandeira, 2013) e as mudanças que ocorreram foram de troca de palavras, mantendo o mesmo sentido nas frases. Da escala a partir da qual foi adaptada (Machado \& Bandeira, 2013), a versão para adolescentes, nomeada Escala de Depressão, Ansiedade e Estresse para Adolescentes (EDAE-A), sofreu as seguintes modificações: as instruções, em escala Likert de 0 a 3 pontos (0 não se aplicou a mim de forma alguma a 3 - aplicou-se muito a mim) foram substituídas por (0 - Não aconteceu comigo nessa semana a 3-Aconteceu comigo na maior parte do tempo da semana). Nas afirmativas, algumas delas sofreram pequenas modificações a fim de facilitar o entendimento dos adolescentes, são elas: a afirmativa 14 - "Eu não tive paciência com nada que interrompesse o que eu estava fazendo" foi substituída por "Eu não tive paciência com coisas que interromperam o que estava fazendo"; a afirmativa 16 - "Não consegui me empolgar com qualquer coisa" foi substituída por "Não consegui me empolgar com nada"; a afirmativa 19 - "Eu percebi as batidas do meu coração na ausência de esforço físico (por exemplo, a sensação de aumento dos batimentos cardíacos, ou de que o coração estava batendo fora do ritmo)" foi substituída por "Eu percebi as batidas do meu coração mais aceleradas sem ter feito esforço físico (por exemplo, a sensação de aumento dos batimentos cardíacos, ou de que o coração estava batendo fora do ritmo)". Por fim, a afirmativa 20 - "Eu me senti assustado(a) sem qualquer razão" foi substituída por "Eu me senti assustado(a) sem ter motivo". Todas as substituições foram realizadas a partir das sugestões dos adolescentes, na aplicação do estudo piloto. Foi obtida autorização do autor da escala original (Lovibond \& Lovibond, 1995) para a realização das modificações propostas, além dos autores que desenvolveram a adaptação para adultos brasileiros (Machado \& Bandeira, 2013).

Após as modificações realizadas, a EDAE-A foi novamente aplicada, em oito adolescentes de 16 a 18 anos, de ambos os sexos, de uma escola de periferia da cidade. Todos os adolescentes responderam, sem dificuldades, à escala e à ficha de dados sociodemográficos. A partir disso, aplicou-se a escala, juntamente com outros instrumentos, a uma amostra final de 426 adolescentes, de 12 a 18 anos, de cinco escolas públicas da cidade de Porto Alegre - RS.

\section{Análise dos Dados}

Para testar o ajuste dos modelos dimensionais concorrentes para a EDAE-A, foram conduzidas análises fatoriais confirmatórias. A matriz de correlações policóricas dos itens foi submetida ao método de estimação Weighted Least Squares Mean - and Variance-adjusted, (WLSMV) (Muthén, Du Toit, \& Spisic, 1997), utilizando-se o software Mplus 6.12 (Muthén \& Muthén, 2010). Os índices de ajuste considerados foram: Comparative Fit Index e Tukey-Lewis Index (CFI e TLI, $\geq 0,95$ ), Root Mean Square Error of Approximation (RMSEA, $\leq$ 0,06 ou $\leq 0,08$ com intervalo de confiança de $90 \%$ ) e a 
significância estatística do teste de Qui-quadrado $(p \geq$ $0,05)$. A consistência interna dos fatores foi calculada pelo método de fidedignidade composta, considerando as cargas fatoriais padronizadas dos itens. Além disso, testes t de Student para amostras independentes foram utilizados a fim de comparar médias no escore total e nas subescalas da EDAE-A entre os sexos e por idade.

\section{Resultados}

Três modelos presentes na literatura foram testados para representar a dimensionalidade da EDAE-A em sua versão para adolescentes. $O$ primeiro compreende apenas um fator geral de afetos negativos $\left[\chi^{2}\right.$ $=645,35$ (189), $p<0,001$, CFI $=0,91$, TLI $=0,90$, RMSEA $=0,075(0,068-0,081)]$. Posteriormente, um modelo de dois fatores foi testado, discriminando a dimensão depressão e uma dimensão de tensão psicológica, reunindo itens de estresse e ansiedade $\left[\chi^{2}=408,97\right.$ (188), $p<0,001$, CFI $=0,95$, TLI $=0,95$, RMSEA $=$ $0,052(0,045-0,059)]$. Por fim, foi investigado o ajuste do modelo original de três fatores representando as dimensões depressão, ansiedade e estresse $\left[\chi^{2}=366,16\right.$ (186), $p<0,001$, CFI $=0,96$, TLI $=0,96$, RMSEA $=$ $0,047(0,040-0,054)]$. Os resultados podem ser observados na Tabela 1. Todas as subescalas demonstraram níveis adequados de consistência interna.

Teste $\mathrm{t}$ de Student para amostras independentes indicou diferença estatisticamente significativa na média da EDAE-A total por sexo, com médias mais altas entre as meninas quando comparadas com os meninos. Também foram observadas diferenças estatisticamente significativas nas médias dos três fatores, tendo as meninas, maior média em todos eles. O mesmo teste foi realizado a fim de comparar se havia diferença nas médias dos escores em cada fator e na média total, por idade - adolescentes mais novos (12 a 15 anos) e adolescentes mais velhos (16 a 18 anos). Não foram observadas diferenças estatisticamente significativas nos grupos em relação às médias em cada fator, nem mesmo no escore total da EDAE-A. Os resultados estão apresentados na Tabela 2.

\section{Discussão}

Este estudo teve por objetivo adaptar e validar a Depression Anxiety and Stress Scale (DASS-21) para adolescentes brasileiros, que recebeu o nome de Escala de Depressão, Ansiedade e Estresse para Adolescentes
(EDAE-A). A partir do instrumento traduzido e validado no Brasil para adultos (Machado \& Bandeira, 2013), poucas foram as modificações realizadas para aplicação do instrumento a adolescentes, referindo-se apenas a aspectos semânticos, conforme necessidade verificada por meio do estudo piloto. Quanto às análises psicométricas do instrumento, a análise fatorial confirmatória corroborou a superioridade do modelo tridimensional, indicando três fatores com bons índices de ajustamento utilizados, sugerindo a adequação dos dados empíricos ao modelo teórico tripartido. Além disso, a confiabilidade - calculada por meio da

Tabela 1

Itens, Cargas Fatoriais e Consistência Interna dos Itens da EDAE- $A$

\begin{tabular}{lccc}
\hline Item & Estresse & Ansiedade & Depressão \\
\hline 1 & 0,74 & & \\
6 & 0,58 & & \\
8 & 0,74 & & \\
11 & 0,55 & & \\
12 & 0,82 & & \\
14 & 0,57 & & \\
18 & 0,73 & & \\
2 & & 0,38 & \\
4 & & 0,65 & \\
7 & & 0,64 & \\
9 & & 0,69 & \\
15 & & 0,77 & \\
19 & & 0,72 & \\
20 & & 0,61 & \\
3 & & & 0,77 \\
5 & & & 0,47 \\
10 & & & 0,73 \\
13 & & & 0,84 \\
16 & & & 0,73 \\
17 & & & 0,83 \\
21 & 0,82 & & 0,86 \\
Ansiedade & & & \\
Depressão & 0,74 & & \\
FC & & & \\
\hline
\end{tabular}

Nota. FC $=$ Fidedignidade Composta, baseada na carga fatorial padronizada dos itens e remete à variância explicada pelo fator. Valores maiores que 0,70 sugerem fidedignidade aceitável. 
Tabela 2

Médias e Desvios Padrão no EDAE-A por Sexo e por Faixa Etária

\begin{tabular}{|c|c|c|c|c|c|c|c|}
\hline & & & Média & $\mathrm{Dp}$ & Teste $t$ & $g l$ & $p$ \\
\hline \multirow{2}{*}{ Depressão } & Sexo & Meninos $(n=162)$ & 3,16 & 3,64 & $-4,38$ & 402 & $<0,001$ \\
\hline & & Meninas $(n=264)$ & 4,95 & 4,73 & & & \\
\hline \multirow{2}{*}{ Ansiedade } & Faixa etária & 12 a 15 anos $(n=256)$ & 4,31 & 4,69 & 0,26 & 424 & 0,79 \\
\hline & & 16 a 18 anos $(n=170)$ & 4,20 & 4,01 & & & \\
\hline \multirow{4}{*}{ Estresse } & Sexo & Meninos $(n=162)$ & 2,29 & 2,62 & $-3,63$ & 415 & $<0,001$ \\
\hline & & Meninas $(n=264)$ & 3,40 & 3,72 & & & \\
\hline & Faixa etária & 12 a 15 anos $(n=256)$ & 2,93 & 3,43 & $-0,37$ & 424 & 0,71 \\
\hline & & 16 a 18 anos $(n=170)$ & 3,06 & 3,33 & & & \\
\hline \multirow{8}{*}{ EDAE-A } & Sexo & Meninos $(n=162)$ & 4,32 & 3,53 & $-5,22$ & 412 & $<0,001$ \\
\hline & & Meninas $(n=264)$ & 6,46 & 4,89 & & & \\
\hline & Faixa etária & 12 a 15 anos $(n=256)$ & 5,60 & 4,64 & $-0,25$ & 424 & 0,89 \\
\hline & & 16 a 18 anos $(n=170)$ & 5,71 & 4,39 & & & \\
\hline & Sexo & Meninos $(n=162)$ & 9,77 & 7,84 & $-5,37$ & 419,7 & $<0,001$ \\
\hline & & Meninas $(n=264)$ & 14,82 & 11,54 & & & \\
\hline & Faixa etária & 12 a 15 anos $(n=256)$ & 12,85 & 11,19 & $-0,12$ & 424 & 0,90 \\
\hline & & 16 a 18 anos $(n=170)$ & 12,97 & 9,58 & & & \\
\hline
\end{tabular}

Nota. EDAE-A: Escala de Depressão, Ansiedade e Estresse para adolescentes

fidedignidade composta - para cada fator indicou bons índices, mostrando que o instrumento está adequado, em termos psicométricos, e pode ser aplicado em adolescentes brasileiros. Esses aspectos demonstram que os sintomas de ansiedade, depressão e estresse são bem discriminados em adolescentes por esse instrumento, reforçando a validade de construto.

Nossas análises confirmam, mas também divergem de alguns estudos prévios realizados em outros países. Por exemplo, este estudo contraria resultados obtidos por Patrick, Dyck e Bramston (2010), que extraíram um fator a partir das análises, indicando que a escala DASS-21 não diferencia sintomas de ansiedade, depressão e estresse em crianças e adolescentes. Já em estudos realizados com adolescentes portugueses (Leal et al., 2009; Pais-Ribeiro et al., 2004), o modelo de três fatores foi superior ao de um ou dois fatores. No entanto, no estudo de Pais-Ribeiro, Honrado e Leal (2004), alguns aspectos apontam para a dificuldade em discriminar os sintomas de depressão, ansiedade e estresse na adolescência. Embora os autores tenham confirmado a superioridade do modelo de três fatores, reconheceram, ao realizar análises de validade convergente e discriminante com outros instrumentos, que as correlações dos itens com as escalas a que não pertencem são mais elevados do que seria o ideal. Esses aspectos não foram avaliados em nosso estudo, sugerindo a necessidade de novos estudos que verifiquem a validade convergente e discriminante da escala EDAE-A com outros instrumentos que se propõem a medir os mesmos construtos.

Neste estudo, apesar de ter encontrado três fatores, eles apresentaram alta correlação entre si, confirmando os resultados do estudo de Pais-Ribeiro et al. (2004). $\mathrm{O}$ estudo desses autores sugere que há contaminação entre os construtos ansiedade, depressão e estresse e que as escalas avaliam aspectos mais semelhantes que diferentes. Esse resultado está de acordo com a teoria subjacente que afirma a associação entre os sintomas das diferentes sintomatologias, embora indique a dificuldade da escala em diferenciar os sintomas de acordo com o modelo tripartido. De fato, esses aspectos revelam a dificuldade em discriminar sintomas de ansiedade e depressão, devido à sobreposição de sintomas associados às duas sintomatologias. Para Pais-Ribeiro et al. (2004), é reconhecida a sobreposição da ansiedade e depressão tanto do ponto de visa da saúde mental quanto do ponto de vista estatístico. 
Neste estudo, não foram observadas cargas fatoriais elevadas em mais de um fator. No entanto, considerou-se as cargas fatoriais com limite inferior aceitável de 0,40 (Hair, Anderson, Tatham, \& Black, 2005). Nesse aspecto, o item 2 apresentou carga baixa $(0,38)$ para ansiedade "Eu percebi que estava com a boca seca". Esse resultado pode ser devido a alguma dificuldade de compreensão do item, o que deverá ser mais bem observado em estudos futuros. Sobre esse aspecto, estudo de Leal et al. (2009) verificou que a consistência interna foi adequada e todos os itens contribuíram para incrementar a consistência. No entanto, após análise dos componentes principais, os autores encontraram que nem todos os itens apresentaram carga adequada na dimensão a que pertenciam. Por outro lado, houve itens com cargas superiores em fatores ao qual não pertenciam originalmente.

Sobre a compreensão dos itens, alguns estudos têm revelado que os adolescentes possuem dificuldades, principalmente em relação aos itens do fator tensão ou estresse, o que não foi verificado neste estudo, talvez devido aos procedimentos de adaptação que evitaram esse tipo de problema. Já no estudo de Szabó (2010), a autora verificou que a DASS-21 fornece evidência inicial para validade de construto em população jovem, no entanto, no geral, o estudo sugere que os adolescentes podem ainda não ter desenvolvido um estado emocional específico semelhante à tensão ou, ainda, esse estado emocional não foi adequadamente avaliado devido à falta de compreensão aos itens do DASS-21. Enquanto os construtos de depressão e ansiedade são semelhantes entre adultos e adolescentes de 11 e 15 anos de idade, a validade da tensão ou estresse é questionável no grupo dos adolescentes (Szabó, 2010). No estudo de Tully, Zajac e Venning (2009), por exemplo, o estresse pareceu ser sinônimo de afeto negativo, sugerindo a necessidade de replicação dos estudos.

Sobre os escores em cada fator e no total, neste estudo foram observadas diferenças significativas nas médias em cada fator (subescala) e no total do escore EDAE-A por sexo, tendo as meninas, maior média em todos eles, quando comparadas com os meninos. Já em relação à faixa etária, adolescentes mais novos $(12$ a 15 anos) e mais velhos (16 a 18 anos) não diferiram. Sobre as diferenças entre os sexos, esse aspecto pode estar relacionado a estereótipos de gênero, que demonstram especificidades quanto à saúde mental das meninas. Para Traverso-Yepez e Pinheiro (2005), na adolescência as meninas percebem mais restrições à liberdade e um aumento de responsabilidades, dúvidas e conflitos, além de maior controle parental - quando comparadas aos meninos - sentindo a adolescência como um período adverso. Sobre esse aspecto, estudos que utilizam outras medidas, como o CDI (Children's Depression Inventory) ou BDI (Beck Depression Inventory), têm encontrado resultados semelhantes que sugerem que o sexo feminino tende a apresentar escores mais altos de sintomas internalizantes quando comparado ao sexo masculino, que tende a apresentar mais sintomas de externalização (Argimon et al., 2013; Azevedo \& Matos, 2014; Hess \& Falcke, 2013). No entanto, os estudos realizados com adolescentes, com o instrumento DASS, não encontraram o mesmo resultado em relação à diferença por sexo. Por exemplo, no estudo de Leal et al. (2009), os autores não encontraram diferenças significativas nos escores da DASS por sexo nem entre as idades de 9 a 11 anos e de 12 a 15 anos. Em outro estudo com adolescentes australianos, diferenças entre os sexos não foram estatisticamente significativas. No entanto, adolescentes mais velhos apresentaram maior média no total dos escores de DASS-21 quando comparados com adolescentes mais novos (Patrick et al., 2010). No estudo de Tully et al. (2009), os meninos, quando comparados com as meninas, apresentaram escores mais elevados para DASS, e em relação à faixa etária; os adolescentes mais velhos apresentaram maiores médias quando comparados aos mais novos. A diversidade de resultados quanto à faixa etária e sexo, nos estudos com a escala DASS em adolescentes, sugere a necessidade de novos estudos.

Sobre a medida de confiabilidade da estrutura fatorial, estes resultados sugerem que a escala EDAE-A apresenta valores que indicam aceitável confiabilidade da estrutura fatorial - 0,86 estresse, 0,83 ansiedade e 0,90 depressão. Esses valores são próximos dos encontrados em outros estudos de validação da escala DASS com adolescentes de outros países. Por exemplo, o estudo de Patrick et al. (2010) indicou valores de 0,97 para depressão; 0,92 para ansiedade e 0,95 para estresse. Já o estudo de Pais-Ribeiro et al. (2004) apresentou valores de 0,85 para a subescala depressão; 0,74 para ansiedade e 0,81 para estresse. O estudo de Leal et al. (2009) indicou boa consistência interna, sendo 0,74 para depressão; 0,78 para ansiedade e 0,75 para estresse.

No geral, verifica-se que, entre os estudos realizados com adolescentes, tanto há divergências nos resultados das análises psicométricas quanto nas comparações dos escores da DASS total e por fatores entre sexo e idade. Esses resultados sugerem a necessidade de mais estudos, principalmente relacionados à validação 
do instrumento com adolescentes. Neste estudo, as análises psicométricas indicam que a EDAE-A é um instrumento bem compreendido pelos estudantes, sendo fácil e rápido em sua aplicação e com propriedades psicométricas satisfatórias, permitindo distinguir sintomas de ansiedade, depressão e estresse. Seu uso pode facilitar o reconhecimento de sintomas que, juntamente com outras ferramentas, pode auxiliar no possível diagnóstico de transtorno mental, essencial para um tratamento e prognóstico adequados.

Sugere-se que novos estudos possam comparar a escala EDAE-A com outros instrumentos que avaliem os mesmos construtos, a fim de atingir uma validade mais completa, já que essa foi uma das limitações deste estudo. Além disso, estudos podem verificar evidências de validade do instrumento em populações de diferentes regiões do país, dada à diversidade do Brasil, além de desenvolver estudos específicos com amostras clínicas. Assim, é importante observar o desempenho do instrumento em diferentes culturas e países. A adaptação em diversos contextos e amostras permite a maior equidade na avaliação. De fato, os estudos transculturais têm atraído a atenção de vários pesquisadores, principalmente na área da saúde mental. Esses estudos permitem verificar diferenças entre indivíduos e culturas, além de possibilitar a compreensão de semelhanças culturais, bem como as diferenças (Borsa, Damásio, \& Bandeira 2012). Dessa forma, sugere-se que novos estudos possam investigar a validade convergente e discriminante da escala com outros instrumentos, além de comparar amostras clínicas com não clínicas e adolescentes de diferentes status socioeconômico.

\section{Referências}

Akin, A., \& Çetin, B. (2007). The Depression Anxiety and Stress Scale (DASS): The study of validity and reliability. Educational Sciences: Theory \& Practice, 7(1), 260-268.

American Psychiatric Association (2014). Manual Diagnóstico e Estatístico de Transtornos Mentais (5 ${ }^{\mathrm{a}} \mathrm{ed}$., rev.). M. Nascimento, Trad. Porto Alegre: Artmed.

Apóstolo, J., Mendes, A., \& Azeredo, Z. (2006). Adaptation to portuguese of the Depression, Anxiety and Stress Scales (DASS). Revista Latino-Americana de Enfermagem, 14(6), 863-871. doi: 10.1590/ S0104-11692006000600006

Argimon, I., Terroso, L., Barbosa, A., \& Lopes, R. (2013). Intensidade de sintomas depressives em adolescentes através da Escala de Depressão de Beck (BDI- II). Boletim Academia Paulista de Psicologia, 33(85), 354-372.

Assis, S. G., Avanci, J. Q., Pesce, R. P., \& Ximenes, L. F. (2009). Situação de crianças e adolescentes brasileiros em relação à saúde mental e à violência. Ciência es Saúde Coletiva, 14(2), 349-361. doi: 10.1590/ S1413-81232009000200002

Azevedo, A., \& Matos, A. (2014). Ideação suicida e sintomatologia depressiva em adolescentes. Psicologia, Saúde e Doenças, 15(1), 180-191. doi: 10.15309/14psd150115.

Bahls, S., \& Bahls, F. (2002). Depressão na adolescência: Características clínicas. Interação em Psicologia, 6(1), 49-57.

Borsa, J., Damásio, B., \& Bandeira, D. R. (2012). Adaptação e validação de instrumentos psicológicos entre culturas: Algumas considerações. Paidéia, 22(53), 423-432. doi: 10.1590/S0103-863X2012000300014

Brasil. (1990). Lei n. 8.069, de 13 de julho de 1990. (1990, 16 de julho). Dispõe sobre o Estatuto da Criança e do Adolescente e dá outras providências. Diário Oficial da União, seção 1.

Brasil (2012). Conselho Nacional de Saúde. Diretrizes e normas para pesquisa envolvendo seres humanos. RESOLUÇÃO Nº 466, de 12 de dezembro de 2012. Brasília: Diário Oficial da União; Poder Executivo, publicado em 13 jun. 2013. Seção I, p.59-62.

Brasil. (2010). Secretaria de Atenção em Saúde. Diretrizes nacionais para a atenção integral à saúde de adolescentes e jovens na promoção, proteção e recuperação da saúde. Brasília: Ministério da Saúde.

Caspi, A., Houts, R., Belsky, D., Goldman-Mellor, S., Harrington, H., Israel, S., Meier, M., Ramrakha, S., Shaley, I., Poulton, R., \& Moffitt, T. (2014). The p factor: One general psychopathology factor in the structure of psychiatric disorders? Clinical Psychological Science, 2(2), 119-137. doi: 10.1177/2167702613497473

Daza, P., Novy, D., Stanley, M., \& Averill, P. (2002). The Depression Anxiety Stress Scale-21: Spanish translation and validation with a Hispanic sample. Journal of Psychopathology \& Behavioral Assessment, 24(3), 195-205. doi: 10.1023/A:1016014818163

Gulliver, A., Griffiths, K., \& Christensen, H. (2010). Perceived barriers and facilitators to

Psico-USF, Bragança Paulista, v. 21, n. 3, p. 459-469, set./dez. 2016 
mental health help-seeking in young people: A sistematic review. BMC Psychiatry, 10, 113-122. doi: 10.1186/1471-244X-10-113.

Hair, J. F., Anderson, R. E., Tatham, R. L., \& Black, W. C. (2005). Análise multivariada de dados. A. S. Sant'Anna \& A. C. Neto (Trans). Porto Alegre: Bookman.

Henry, J. D., \& Crawford, J. R. (2005). The shortform version of the Depression Anxiety Stress scales (DASS-21): Construct validity and normative data in a large non-clinical sample. British Journal of Clinical Psychology, 44(2), 227-239. doi: 10.1348/014466505X29657

Hess, A. R., \& Falcke, D. (2013). Sintomas internalizantes na adolescência e as relações familiars: Uma revisão sistemática da literature. Psico-USF, 18(2), 263-276. doi: 10.1590/S1413-82712013000200010

Jatobá, J., \& Batos, O. (2007). Depressão e ansiedade em adolescentes de escolas públicas e privadas. Jornal Brasileiro de Psiquiatria, 56(3), 171-179. doi: 10.1590/S0047-20852007000300003.

Kieling, C., \& Belfer, M. (2012). Oportunidade e desafio: A situação da saúde mental da infância e adolescência no Brasil. Revista Brasileira de Psiquiatria, 34(3), 241-244. doi: 10.1016/j.rbp.2012.05.003

Leal, I., Antunes, R., Passos, T., Pais-Ribeiro, \& Maroco, J. (2009). Estudo da Escala de Depressão, Ansiedade e Stresse para crianças (EADS-C). Psicologia: Saúde \& Doenças, 10(2), 277-284.

Lovibond, P. F., \& Lovibond, S. H. (1995). The structure of negative emotional states: Comparison of the Depression Anxiety Stress Scales (DASS) with the Beck depression and anxiety inventories. Behaviour Research and Therapy, 33(3), 335-343. doi: 10.1016/j.rbp.2012.05.003

Lyrakos, G. N., Arvaniti, C., Smyrnioti, M., \& Kostopanagiotou, G. (2011). Translation and validation study of the Depression Anxiety Stress Scale in the Greek general population and in a psychiatric patient's sample. European Psychiatry 26, 1731-1731. doi: 10.1016/S0924-9338(11)73435-6

Machado, W. L., \& Bandeira, D. R. (2013). Adaptação e validação da Depression, Anxiety and Stress Scale (DASS-21) para o Português brasileiro. Manuscript submitted for publication.
Musa, R., Fadzil, M., \& Zain, Z. (2007). Translation, validation and psychometric properties of Bahasa Malaysia version of the Depression Anxiety and Stress Scales (DASS). ASEAN Journal of Psychiatry, 8(2), 82-89.

Muthén, B., Du Toit, S. H. C., \& Spisic, D. (1997). Robust inference using weighted least squares and quadratic estimating equations in latent variable modeling with categorical and continuous outcomes. Non-published technical report.

Muthén, L. K., \& Muthén, B. O. (2010). Mplus: Statistical analysis with latent variables. User's guide. Los Angeles: Muthén \& Muthén.

Pais-Ribeiro, J., Honrado, A., \& Leal, I. (2004). Contribuição para o estudo da adaptação portuguesa das Escalas de Ansiedade, Depressão e Estresse (EADS) de 21 itens de Lovibond e Lovibond. Psicologia, Saúde \& Doenças, 5(2), 229-239.

Patrick, J., Dick, M., \& Bramston, P. (2010). Depression Anxiety Stress Scale: Is it valid for children and adolescents? Journal of Clinical Psychology, 66(9), 9961007. doi: $10.1002 /$ jclp.20696.

Pinto, A., Luna, I., Silva, A., Pinheiro, P., Braga, V., \& Souza, A. (2014). Fatores de risco associados a problemas de saúde mental em adolescentes: Revisão Integrativa. Revista Escola de Enfermagem, 48(3), 555564. doi: 10.1590/S0080-623420140000300022

Rocha, T., Zeni, C., Caetano, S., \& Kieling, C. (2013). Mood disorders in childhood and adolescence. Revista Brasileira de Psiquiatria, 35, 22-31. doi: 10.1590/1516-4446-2013-S106

Rosa, A., Loureiro, L., \& Sequeira, C. (2014). Literacia em saúde mental de adolescentes: Um estudo exploratório. Revista Portuguesa de Enfermagem de Saúde Mental, $n^{\circ}$ especial, 125-132.

Szabó, M. (2010). The short version of the Depression Anxiety Stress Scales (DASS-21): Factor structure in a young adolescent sample. Journal of Adolescence, 33, 1-8. doi: 0.1016/j.adolescence.2009.05.014.

Thapar, A., Collishaw, S., Pine, D., \& Thapar, A. K. (2012). Depression in adolescence. Lancet, 379, 1056-1067. doi: 10.1016/S0140-6736(11)60871-4

Traverso-Yepez, M. A., \& Pinheiro, V. S. (2005). Socialização de gênero e adolescência. Estudos Feministas, 13(1), 147-162. doi: 10.1590/ S0104-026X2005000100010 
Tully, P., Zajac, I., \& Venning, A. (2009). The structure of anxiety and depression in a normative sample of younger and older Australian adolescents. Journal of Abnormal Child Psychology, 37, 717-726. doi: 10.1007/s10802-009-9306-4

Vignola, R., \& Tucci, A. (2014). Adaptation and validation of the Depression Anxiety and Stress Scale (DASS) to Brazilian Portuguese. Journal of Affective Disorders, 155, 104-109. doi: 10.1016/j. jad.2013.10.031

Watson, D., Weber, K., Assenheimer, J., Clark, L., Strauss, M., \& McCormick, R. (1995). Testing a tripartite model: I. Evaluating the convergent and discriminant validity of Anxiety and Depression Symptom Scales, Journal of Abnormal Psychology, 104(1), 3-14. doi: 10.1037/0021-843X.104.1.3

World Health Organization (2012). Adolescent mental health: Mapping actions of nongovernmental organizations and other international development organizations. Geneva: Word Health Organization. Recuperado de http://www.who.int/mental_health/publications/adolescent_mental_health/en/index.html

Recebido 20/04/2015

Reformulado 09/06/2015

Aceito 21/08/2015

Sobre os autores:

Naiana Dapieve Patias é psicóloga, mestre em Psicologia (UFSM) e doutora em Psicologia (UFRGS). Docente do curso de graduação e Pós-Graduação em Psicologia (IMED).

E-mail: naipatias@hotmail.com

Wagner de Lara Machado é psicólogo, mestre e doutor em Psicologia (UFRGS) e docente da Pontifícia Universidade Católica de Campinas (PUC-Campinas).

E-mail:wag_psico@yahoo.com.br

Denise Ruschel Bandeira é psicóloga, mestre e doutora em Psicologia (UFRGS), docente do Programa de Pós-Graduação em Psicologia (UFRGS).

E-mail: deniserbandeira@gmail.com

Débora Dalbosco Dell'Aglio é psicóloga, mestre e doutora em Psicologia (UFRGS) e docente do Programa de Pós-Graduação em Psicologia (UFRGS).

E-mail: debora.dellaglio@ufrgs.br

Contato com os autores:

Naiana Dapieve Patias

Faculdade Meridional (IMED)

Rua Senador Pinheiro, 304. Sala dos Professores

CEP: 99070-220

Passo Fundo-RS, Brasil 


\section{Anexo A}

Escala de Depressão, Ansiedade e Estresse para Adolescentes (EDAE-A)

Por favor, leia cada afirmativa e marque um dos números $(0,1,2$, ou 3$)$ que indique quanto a afirmativa aconteceu a você na última semana. Não há respostas certas ou erradas. Não gaste muito tempo em nenhuma das afirmativas

0 Não aconteceu comigo nessa semana

1 Aconteceu comigo algumas vezes na semana

2 Aconteceu comigo em boa parte da semana

3 Aconteceu comigo na maior parte do tempo da semana

1 Eu tive dificuldade para me acalmar

2 Eu percebi que estava com a boca seca

3 Eu não conseguia ter sentimentos positivos

$4 \quad$ Eu tive dificuldade para respirar

(por exemplo, tive respiração muito rápida, ou falta de ar sem ter feito esforço físico)

$5 \quad$ Foi difícil ter iniciativa para fazer as coisas

6 Em geral, tive reações exageradas às situações

7 Tive tremores (por exemplo, nas mãos)

8 Eu senti que estava bastante nervoso(a)

9 Eu fiquei preocupado(a) com situações em que poderia entrar em pânico e fazer papel de bobo(a)

10 Eu senti que não tinha expectativas positivas a respeito de nada

11 Notei que estava ficando agitado(a)

12 Achei difícil relaxar

13 Eu me senti abatido(a) e triste

14 Eu não tive paciência com coisas que interromperam o que estava fazendo

15 Eu senti que estava prestes a entrar em pânico

16 Não consegui me empolgar com nada

17 Eu senti que não tinha muito valor como pessoa

18 Eu senti que eu estava muito irritado(a)

19 Eu percebi as batidas do meu coração mais aceleradas sem ter feito esforço físico (por exemplo, a sensação de aumento dos batimentos cardíacos, ou de que o coração estava batendo fora do ritmo)

20 Eu me senti assustado(a) sem ter motivo

$\begin{array}{llll}0 & 1 & 2 & 3\end{array}$

$\begin{array}{llll}0 & 1 & 2 & 3\end{array}$

$\begin{array}{llll}0 & 1 & 2 & 3\end{array}$

$\begin{array}{llll}0 & 1 & 2 & 3\end{array}$

$\begin{array}{llll}0 & 1 & 2 & 3\end{array}$

21 Eu senti que a vida não tinha sentido

\section{Permissão para o uso do instrumento}

Não há necessidade de solicitar permissão para o uso do instrumento. No entanto, é exigido que o devido crédito seja concedido aos seus autores. O presente artigo deve ser utilizado para a citação do instrumento, e esta nota assegura a permissão para sua utilização. 